\title{
Drifting Breathers and Fermi-Pasta-Ulam Paradox for Water Waves
}

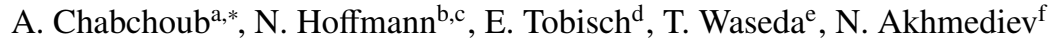 \\ ${ }^{a}$ Centre for Wind, Waves and Water, School of Civil Engineering, The University of Sydney, Sydney, NSW 2006, Australia \\ ${ }^{b}$ Dynamics Group, Hamburg University of Technology, 21073 Hamburg, Germany \\ ${ }^{c}$ Department of Mechanical Engineering, Imperial College London, London SW7 2AZ, United Kingdom \\ ${ }^{d}$ Institute for Analysis, Johannes Kepler University, 4040 Linz, Austria \\ ${ }^{e}$ Department of Ocean Technology Policy and Environment, Graduate School of Frontier Sciences, The University of Tokyo, Kashiwa, Chiba \\ 277-8563, Japan \\ ${ }^{f}$ Research School of Physics and Engineering, The Australian National University, Canberra, ACT 2000, Australia
}

\begin{abstract}
One physical mechanism that is responsible for the focusing of uni-directional water waves is the modulation instability (MI). This occurs when side-bands around the main frequency are excited either deterministically or randomly and subsequently grow exponentially. In physical space the periodically-perturbed wave group can reach significant wave amplifications and in the case of infinite modulation period even three times the initial amplitude of the regular Stokes wave train. These periodic wave groups propagate in deep-water with a group velocity half the wave's phase speed. In this experimental study, we investigate the dynamics of modulationally unstable wave groups that propagate with a velocity that is higher than the packet's group velocity in deep-water. It is shown that when this additional velocity to the wave group is marginal, a very good agreement with nonlinear Schrödinger (NLS) hydrodynamics is reached at all stage of propagation and the characteristic wave energy cascade remains symmetric and stationary. Otherwise, a significant deviation is observed only at the stage of large wave focusing of the isolated wave packet. In this case, the wave field experiences an almost perfect return to the initial conditions after the compression, a particular dynamics also known as the Fermi-Pasta-Ulam paradox.
\end{abstract}

Keywords: Nonlinear waves, Breathers, Nonlinear Dynamics

\section{Introduction}

Uni-directional water waves can focus as a result of the Benjamin-Feir instability, also referred to as modulation instability (MI) in a more general context $[1,2,3]$. The focusing dynamics of the latter is different from the dispersive focusing or superposition of waves [4]. It is indeed important to segregate both mechanisms, particularly, when breaking results from severe wave steepening $[5,6,7,8,9]$. It is also well-known that MI can also describe localized patterns in other dispersive media, for instance in optics [10, 11].

One elegant methodology to investigate the MI in laboratory environments is through exact solutions of the nonlinear Schrödinger equation (NLS) [2, 12]. As exact solutions provide a deterministic description of weakly

\footnotetext{
${ }^{*}$ Corresponding author

Email address: amin. chabchoub@sydney.edu.au (A. Chabchoub)
}

Preprint submitted to Wave Motion nonlinear water wave patterns in time and space, boundary conditions can be easily determined to initiate the wave dynamics of interest by a wave generator. The elementary solution that describes the classical MI wave motion is known as Akhmediev breather (AB) [13]. It is a one parameter family of solutions in which the free parameter determines the unstable modulation frequency that corresponds to the MI exponential growth rate. Note that the solution also describes the fundamental Fermi-Pasta-Ulam (FPU) paradox [14] since the dynamics is initiated and terminated being described by a three wave system $[3,15]$. In the limit of infinite modulation period, this latter periodic solution converges to a doubly-localized breather, also known as the Peregrine soliton [16, 17, 18, 19]. As advanced model for MI, the drifting $\mathrm{AB}$ models incorporate a second parameter that enables to determine an angle between the line of maxima of the envelope and the temporal axis in the framework moving with the group velocity [20]. As a result, periodic unstable wave packets are expected to 
propagate with a velocity that is higher than the group velocity $[21,20]$.

In this work, we report and discuss experiments on drifting and unstable hydrodynamic wave packets within the context of the drifting AB models. The laboratory measurements show a very good agreement with the NLS prediction, especially, when the additional drift speed is marginal. The spectral analysis reveals that the characteristic side-band cascades remain symmetric and stationary throughout the propagation while a FPU paradox and an asymmetry of the focused wave spectra is noticeable for large breather drift values.

\section{Drifting Akhmediev breathers}

The time-NLS is a fundamental framework $[2,22]$ that describes the evolution of weakly nonlinear deepwater wave packets $\psi(x, t)$ evolving along the spatial co-ordinate $x$

$$
\mathrm{i}\left(\psi_{x}+\frac{1}{c_{g}} \psi_{t}\right)-\frac{1}{g} \psi_{t t}-k^{3}|\psi|^{2} \psi=0
$$

These wave packets propagate with the group velocity $c_{g}=\omega /(2 k)$, while $k$ and $\omega$ are the wave number and wave frequency, respectively. The NLS admits a plane wave solution that corresponds to a second-order Stokes wave $[23,24,12]$. The exact model that describes the dynamics of modulationally unstable Stokes waves in time and space is referred to as $\mathrm{AB}$ [25]. Considering the following scaled form to the NLS

$$
\mathrm{i} q_{X}+q_{T T}+2|q|^{2} q=0,
$$

the deterministic expression for periodic as well as drifting unstable Stokes envelopes $\psi_{D A B}$ are given by [20]:

$$
\psi_{D A B}(X, T)=\left(\frac{G+\mathrm{i} H}{D}\right) \exp (2 \mathrm{i} X)
$$

where

$$
\begin{aligned}
G= & 2 \cosh \left[\kappa_{i} T+2\left(\kappa_{r} l_{i}+\kappa_{i} l_{r}\right) X\right] \\
& \times\left[\cosh \left(2 \chi_{i}\right)-2 l_{i} \sin \left(2 \chi_{r}\right)\right] \\
+ & 2 \cos \left[2 \kappa_{i} l_{i} X-\kappa_{r}\left(T+2 l_{r} X\right)\right] \\
& \times\left[2 l_{i} \cosh \left(2 \chi_{i}\right)-\sin \left(2 \chi_{r}\right)\right], \\
H=\quad 4 & l_{i}\left\{\cos \left(2 \chi_{r}\right) \sinh \left[\kappa_{i} T+2\left(\kappa_{r} l_{i}+\kappa_{i} l_{r}\right) X\right]\right. \\
& \left.-\sin \left[2 \kappa_{i} l_{i} X-\kappa_{r}\left(T+2 l_{r} X\right)\right] \sinh \left(2 \chi_{i}\right)\right\}, \\
D= & 2 \cosh \left(\kappa_{i} T+2 \kappa_{r} l_{i} X+2 \kappa_{i} l_{r} X\right) \cosh \left(2 \chi_{i}\right) \\
& -2 \cos \left(\kappa_{r} T-2 \kappa_{i} l_{i} X+2 \kappa_{r} l_{r} X\right) \sin \left(2 \chi_{r}\right),
\end{aligned}
$$

Complex coefficients $\chi$ and $\kappa$ here are given by

$$
\chi=\frac{1}{2} \arccos (\kappa / 2), \quad \kappa=\sqrt{1+l^{2}}
$$

with $l=l_{r}+\mathrm{i} l_{i}$ being the complex eigenvalue of the inverse scattering technique. The subscripts $r$ and $i$ in the complex coefficients $\chi$ and $\kappa$ denote the real and imaginary parts, respectively.

When $l_{r}=0$, the expression (3) is the well known zero-velocity AB [20]. For non-zero $l_{r}$, the breather acquires the drift with the velocity in $(X, T)$-plane equal to

$$
V=\frac{\kappa_{i}}{2\left(\kappa_{r} l_{i}+\kappa_{i} l_{r}\right)} \text {. }
$$

As we can see, both parameters $l_{r}$ and $l_{i}$ control the drift speed of the AB. Clearly, when $l_{r}=0$, velocity becomes zero, $V=0$. Interestingly, nonzero velocity leads to extended range of existence of the ABs. Namely, the range of $l_{i}$-values that admits the existence of ABs becomes much wider.

Two examples of drifting ABs are depicted in Fig. 1. The velocity of the $A B$ on the left is higher than the velocity of the $\mathrm{AB}$ on the right, in agreement with the equation (4). The figure also shows that for a fixed value of $l_{r}$, the drift $V$ is higher when increasing the value of $l_{i}$. The maximal expected amplitude amplification of three in the NLS context is reached, when $l_{i}=1$ for an arbitrary choice of $l_{r}$.
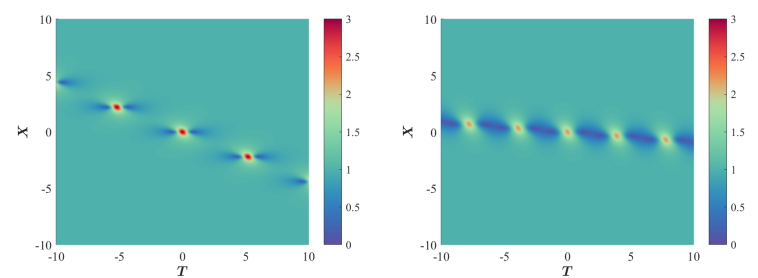

Figure 1: (Left) Amplitude-normalized Drifting AB for $l=0.12+1 \mathrm{i}$. (Right) Amplitude-normalized drifting $\mathrm{AB}$ for $l=0.12+0.6 \mathrm{i}$

The presence of the drift allows to collide ABs the same way as solitons. Using the techniques of inverse scattering, the solution (3) can be further extended to describe collision and nonlinear interactions of two and more ABs (see the works [20, 26, 27] for further details).

\section{Experimental set-up}

The experiments have been conducted in a large wave basin that is installed at The University of Tokyo. It has the dimensions $50 \times 10 \times 5 \mathrm{~m}^{3}[28,29]$. The eleven wave 

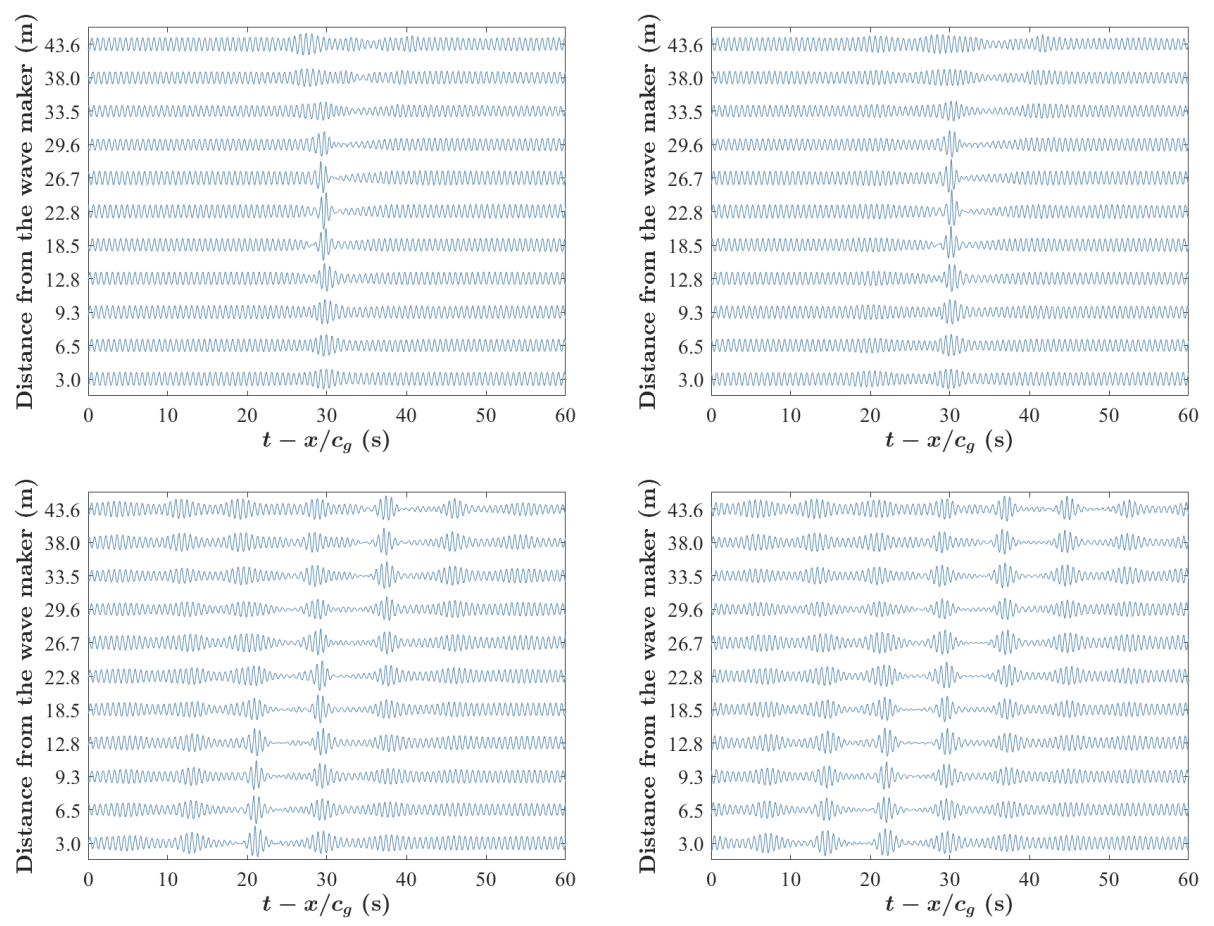

Figure 2: Evolution of four different drifting ABs for the carrier parameters $a=0.01 \mathrm{~m}$ and $a k=0.08$ with same drift parameters, however, different modulation frequencies. (Top left) $l_{r}=0.12$ and $l_{i}=1$ (maximal amplitude amplification). (Top right) $l_{r}=0.12$ and $l_{i}=0.9$. (Bottom left) $l_{r}=0.12$ and $l_{i}=0.7$. (Bottom right) $l_{r}=0.12$ and $l_{i}=0.6$.

wires at $3.1 \mathrm{~m}, 6.6 \mathrm{~m}, 9.38 \mathrm{~m}, 13.2 \mathrm{~m}, 18.5 \mathrm{~m}, 22.8 \mathrm{~m}$, $26.7 \mathrm{~m}, 29.6 \mathrm{~m}, 33.6 \mathrm{~m}, 38.0 \mathrm{~m}$ and $43.6 \mathrm{~m}$, respectively, record the water surface elevation measurements with a sampling frequency of $100 \mathrm{~Hz}$. The boundary conditions that define the wave maker's motion to observe the drifting breathers is determined through the dimensional and uni-directional surface elevation, to firstorder of approximation

$$
\eta(x, t)=\operatorname{Re}\left(\psi_{D A B}(x, t) \exp [\mathrm{i}(k x-\omega t)]\right)
$$

by fixing the space co-ordinate at the position $x^{*}=-20$ $\mathrm{m}$. As such, the maximal breather compression is expected to occur $20 \mathrm{~m}$ from the wave maker. The carrier wave parameters have been set to have an amplitude of $a=0.01 \mathrm{~m}$ and a wave steepness of $a k=0.08$ for all runs. This corresponds to a frequency value of $f=\frac{\omega}{2 \pi}=1.40 \mathrm{~Hz}$. The breather transformations to dimensional units are straight-forward $[30,12]$. Note that when validating the data, one has to take into account the second-order Stokes correction, that is

$$
\begin{aligned}
\eta(x, t)= & \frac{1}{2}\left(\psi_{D A B}(x, t) \exp [\mathrm{i}(k x-\omega t)]\right. \\
& +\frac{1}{2} k \psi_{D A B}^{2}(x, t) \exp [2 \mathrm{i}(k x-\omega t)] \\
& + \text { c.c. }),
\end{aligned}
$$

c.c. denotes the complex conjugate.

\section{Experimental results}

In this section, we describe and analyze the evolution of different drifting $\mathrm{AB}$ solutions. We recall that the carrier parameters, that is, the background amplitude and steepness are the same in all experimental cases. In fact, only the modulation frequency parameter (imaginary part of the parameter $l$ ) and the drift parameter (real part of the parameter $l$ ) have been varied.

First, we show the evolution of four different drifting ABs with parameter pairs $l_{r}=0.12$ and $l_{i}=1, l_{r}=0.12$ and $l_{i}=0.9, l_{r}=0.12$ and $l_{i}=0.7$ as well as $l_{r}=0.12$ and $l_{i}=0.6$. All four cases are shown in Fig. 2 .

We can qualitatively compare the wave motion with the theoretical expectations for each case. These are shown in Fig. 3. 

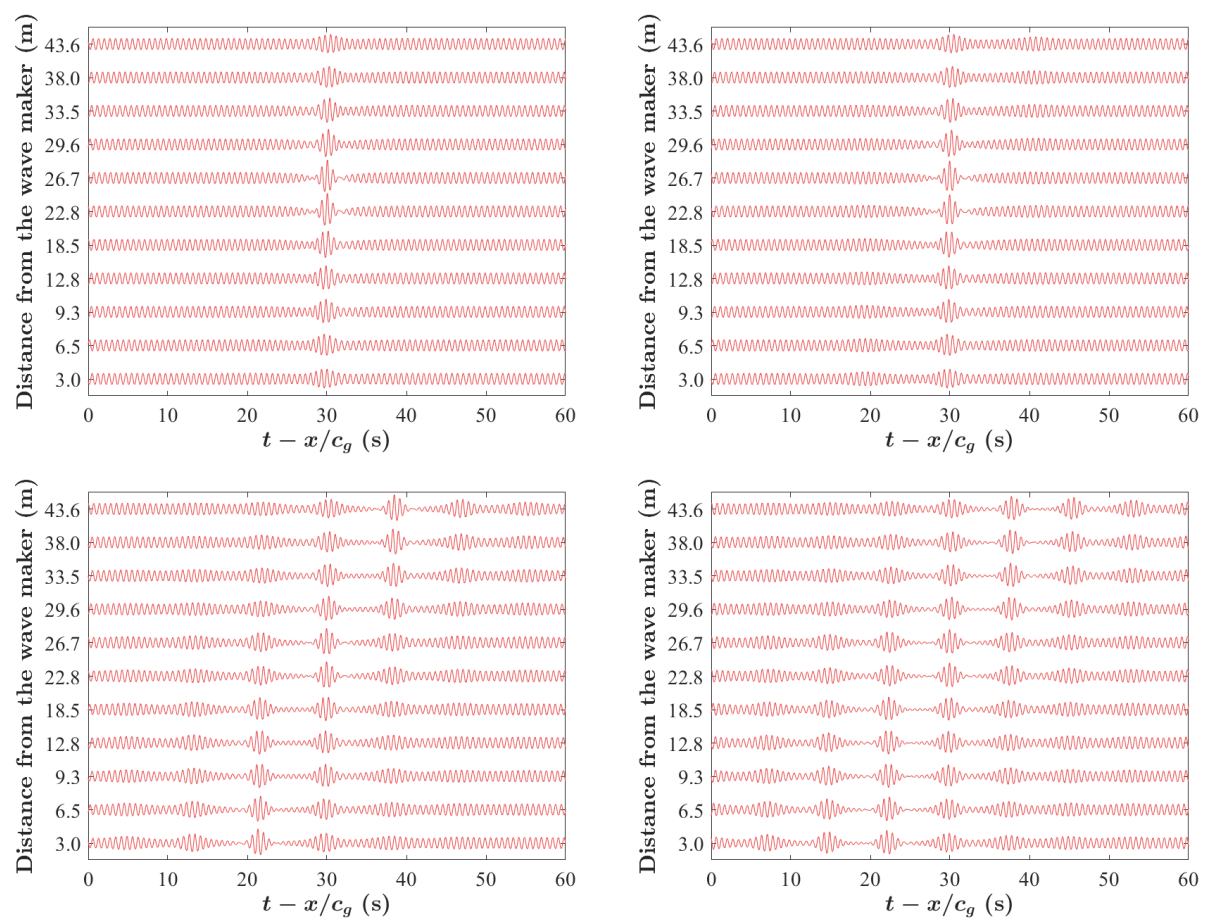

Figure 3: NLS prediction of the wave dynamics observed in Fig. 2 of four different drifting ABs for the carrier parameters $a=0.01 \mathrm{~m}$ and $a k=0.08$ with same drift parameters, however, different modulation frequencies. (Top right) $l_{r}=0.12$ and $l_{i}=1$ (maximal amplitude amplification). (Bottom left) $l_{r}=0.12$ and $l_{i}=0.9$. (Right left) $l_{r}=0.12$ and $l_{i}=0.7$. (Top left) $l_{r}=0.12$ and $l_{i}=0.6$.

Indeed, the higher the value of the parameter $l_{i}$, the smaller the modulation frequency and as such, the higher the focusing amplitude. In the first two experiments, we can clearly note the asymmetry of the wave packets when the almost single breather is significantly compressed. This is known to be the consequence of higher-order effects not captured by the NLS, when extreme wave steepening takes place. These corrections can be described by the modified NLS [31, 32, 33, 34], that is the more accurate framework describing such steep waves. In the cases of marginal drift speed, the wave packets remain indeed symmetric at all stages of evolution. Moreover, the shape of the unstable envelopes in the latter cases resembles the propagation of the ABs within the framework of the space-NLS (the framework evolving the wave packets along the temporal co-ordinate $t$ ) $[35,36,37]$.

It is well-known that the MI and the standard ABs are connected to the FPU paradox, since the focusing dynamics in an idealized case is initiated and completed by three waves (carrier frequency and a side-band pair) $[38,39]$. The initial and final stage of wave focusing is expected to be identical for the drifting ABs as well, however, the shape of the spectra is indeed different and evolves differently from case to case, particularly, when more than one side-bands are initially involved in the dynamics. As next, we illustrate in Fig. 4 the initial, mid-stage (maximal breather compression) and final wave spectra of the wave evolution of the cases already described in Fig. 2 and 3.

We can deduct from Fig. 4 that the more side-bands initially triggered (that is, the smaller values of $V$ ), the more stationary the spectral evolution is expected to be. This is eminent for the breather parameters $l_{r}=0.12$ and $l_{i}=0.6$. On the other hand, the higher the value of $V$, the less side-bands are initially triggered. In the latter case, the wave field experiences an asymmetry during focusing. Nevertheless, a return to the initial conditions can be observed and as such, a FPU paradox dynamics.

Based on these characteristic breather features, it becomes obvious that such waves may have relevant implication for potential applications, particularly, when investigating the interaction of nonlinear and extreme waves with marine structures [40]. In fact, by varying the modulation frequency and drift speed, we can either model one single extreme localization or different extreme wave groups that gradually increase in wave amplitude at a specific location. 

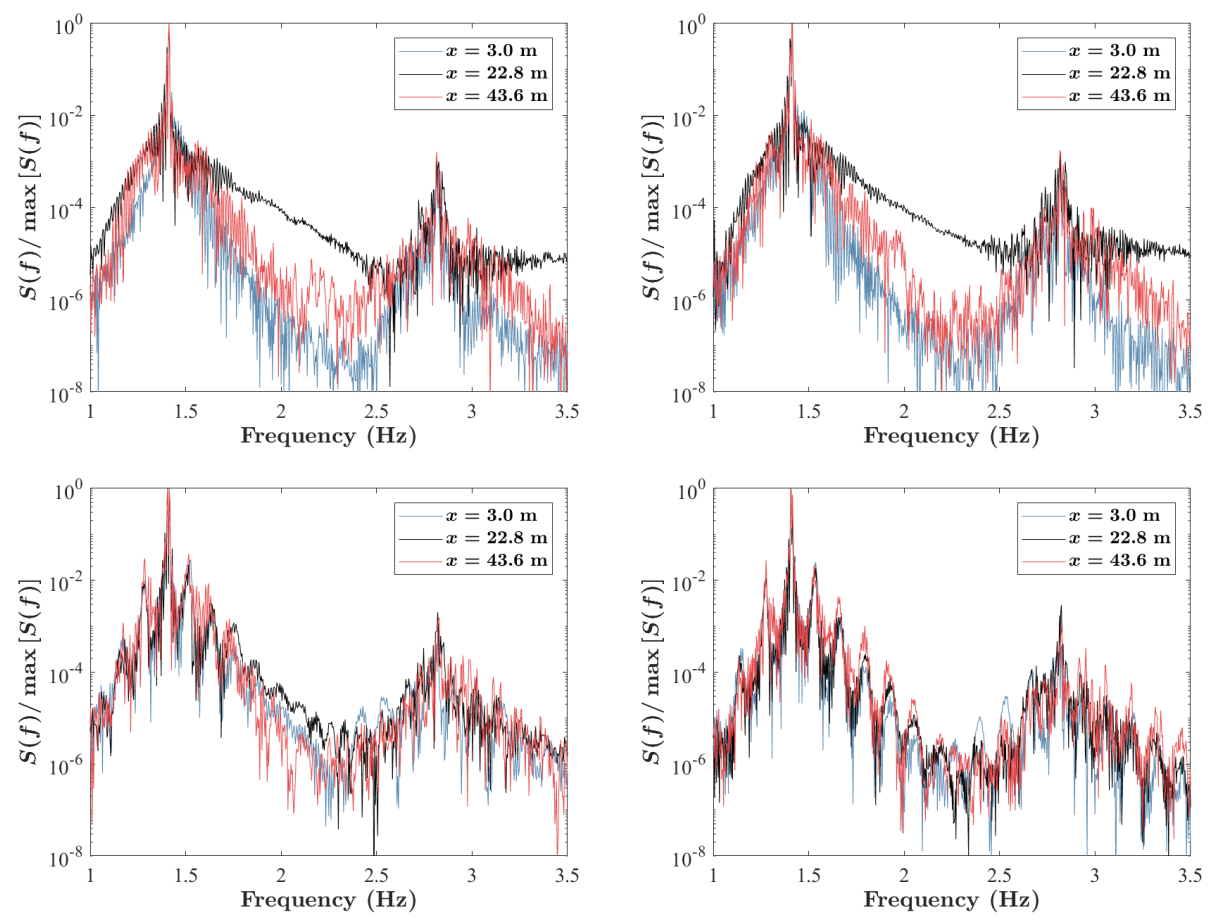

Figure 4: The normalized power spectra corresponding to the rogue packet evolution cases, shown in Fig. 2 for the measurements at $3.1 \mathrm{~m}$ (blue lines), $22.8 \mathrm{~m}$ (black lines) and $43.6 \mathrm{~m}$ (red lines).

Moreover, we emphasize that spectral downshifting was not observed in the experiments neither at the stage of maximum focusing neither after decompression of the breather. This suggests that wave breaking or forcing effects are not present in the reported experiments [5].

\section{Conclusion}

We investigated experimentally the dynamics of drifting modulationally unstable wave groups within the framework of exact solutions of the hydrodynamic NLS, known as drifting ABs models. These NLS solutions have two parameters that control the modulation frequency and the drift speed of the unstable wave packets. The surface water wave profiles collected in the wave flume for the same background parameters reveal that when one single drifting and isolated extreme localization is modelled (modulation frequency close to zero) red, a return to the initial conditions is qualitatively observed even though experiencing a noticeable asymmetry when focusing. However, this latter discrepancy does not occur when the modulation frequency admits higher values and for the case of marginal drift speed. Furthermore, we have shown that the drifting
ABs describe different types of FPU scenario with different side-band pair as initial and last stage of wave focusing, before the focusing cycle recommences. Furthermore, downshifting has not been observed for all experiments.

An interesting point is that modulation instability triggers an energy transport scenario in the wave systems with moderate nonlinearity. This process can be described using the ideas of dynamical D-cascade [41]. Namely, modes in the D-cascade can be related to spectral components of the fundamental $\mathrm{AB}$. Using this approach allows one to calculate the amplitude and the wave steepness of the carrier wave, the asymmetry of the spectra and the shape of the energy spectra. Moreover, conditions for D-cascade stabilization can be found. The method also defines the number of modes in the direct and inverse cascades of the Fourier space. The appearance of the FPU-like recurrence can also be explained [42]. It might be an interesting task to simplify the model of the D-cascade using a single drifting $\mathrm{ABs}$ rather than a set of fundamental ABs.

Future work will be also devoted to investigate the corresponding drift and FPU mechanisms in the fully nonlinear water wave context $[43,44]$. 


\section{Acknowledgments}

The laboratory experiments have been conducted under support of the Japan Society for Promoting Science (JSPS), Grant-in-Aid for Scientific Research. A.C. is thankful to the members of the WasedaLab (The University of Tokyo) for the help in setting up the experiments.N.H. acknowledges the support from the Deutsche Forschungsgemeinschaft (DFG) under the project Ho 3852-10/1. E.T. is thankful to the support by the Austrian Science Foundation (FWF) under project P30887. N.A. acknowledges the support of the Australian Research Council (Discovery Project DP150102057) and support from the Volkswagen Stiftung.

\section{References}

[1] T. B. Benjamin, J. Feir, The disintegration of wave trains on deep water Part 1. Theory, Journal of Fluid Mechanics 27 (03) (1967) 417-430.

[2] V. E. Zakharov, Stability of periodic waves of finite amplitude on the surface of a deep fluid, Journal of Applied Mechanics and Technical Physics 9 (2) (1968) 190-194.

[3] M. P. Tulin, T. Waseda, Laboratory observations of wave group evolution, including breaking effects, Journal of Fluid Mechanics 378 (1999) 197-232.

[4] T. A. Adcock, P. H. Taylor, The physics of anomalous (rogue) ocean waves, Reports on Progress in Physics 77 (10) (2014) 105901.

[5] M. P. Tulin, Breaking of ocean waves and downshifting, in: Waves and Nonlinear Processes in Hydrodynamics, Springer, 1996, pp. 177-190.

[6] A. Babanin, Breaking and dissipation of ocean surface waves, Cambridge University Press, 2011.

[7] M. Onorato, D. Proment, G. Clauss, M. Klein, Rogue waves: from nonlinear Schrödinger breather solutions to sea-keeping test, PloS one 8 (2) (2013) e54629.

[8] A. Alberello, A. Chabchoub, J. P. Monty, F. Nelli, J. H. Lee, J. Elsnab, A. Toffoli, An experimental comparison of velocities underneath focussed breaking waves, Ocean Engineering 155 (2018) 201-210.

[9] M. Derakhti, M. L. Banner, J. T. Kirby, Predicting the breaking strength of gravity water waves in deep and intermediate depth, Journal of Fluid Mechanics 848.

[10] M. Onorato, S. Residori, U. Bortolozzo, A. Montina, F. Arecchi, Rogue waves and their generating mechanisms in different physical contexts, Physics Reports 528 (2) (2013) 47-89.

[11] J. M. Dudley, F. Dias, M. Erkintalo, G. Genty, Instabilities, breathers and rogue waves in optics, Nature Photonics 8 (10) (2014) 755-764.

[12] A. Osborne, Nonlinear Ocean Waves and the Inverse Scattering Transform, Int, Vol. 97, 2010.

[13] N. Akhmediev, V. Eleonskii, N. Kulagin, Generation of a periodic sequence of picosecond pulses in an optical fiber-Exact solutions, Zhurnal Eksperimentalnoi i Teoreticheskoi Fiziki 89 (1985) 1542-1551.

[14] E. Fermi, P. Pasta, S. Ulam, M. Tsingou, Studies of the nonlinear problems, Tech. rep., Los Alamos Scientific Lab., N. Mex. (1955).
[15] J. M. Dudley, G. Genty, F. Dias, B. Kibler, N. Akhmediev, Modulation instability, Akhmediev breathers and continuous wave supercontinuum generation, Optics Express 17 (24) (2009) 21497-21508.

[16] D. Peregrine, Water waves, nonlinear Schrödinger equations and their solutions, The Journal of the Australian Mathematical Society. Series B. Applied Mathematics 25 (01) (1983) 16-43.

[17] B. Kibler, J. Fatome, C. Finot, G. Millot, F. Dias, G. Genty, N. Akhmediev, J. M. Dudley, The Peregrine soliton in nonlinear fibre optics, Nature Physics 6 (10) (2010) 790-795.

[18] A. Chabchoub, N. Hoffmann, N. Akhmediev, Rogue wave observation in a water wave tank, Physical Review Letters 106 (20) (2011) 204502.

[19] A. Chabchoub, Tracking breather dynamics in irregular sea state conditions, Physical Review Letters 117 (14) (2016) 144103.

[20] N. Akhmediev, J. M. Soto-Crespo, A. Ankiewicz, Extreme waves that appear from nowhere: on the nature of rogue waves, Physics Letters A 373 (25) (2009) 2137-2145.

[21] M. Tajiri, Y. Watanabe, Breather solutions to the focusing nonlinear schrödinger equation, Physical Review E 57 (3) (1998) 3510 .

[22] A. Shabat, Exact theory of two-dimensional self-focusing and one-dimensional self-modulation of waves in nonlinear media, Soviet Physics JETP 34 (1) (1972) 62.

[23] G. G. Stokes, On the theory of oscillatory waves, Trans Cambridge Philos Soc 8 (1847) 441-473.

[24] H. C. Yuen, B. M. Lake, Nonlinear dynamics of deep-water gravity waves, Advances in Applied Mechanics 22 (1982) 67229.

[25] N. Akhmediev, V. Eleonskii, N. Kulagin, Exact first-order solutions of the nonlinear Schrödinger equation, Theoretical and Mathematical Physics 72 (2) (1987) 809-818.

[26] N. Akhmediev, J. M. Soto-Crespo, A. Ankiewicz, How to excite a rogue wave, Physical Review A 80 (4) (2009) 043818.

[27] B. Frisquet, B. Kibler, G. Millot, Collision of Akhmediev breathers in nonlinear fiber optics, Physical Review X 3 (4) (2013) 041032

[28] M. J. Mozumi, T. Waseda, A. Chabchoub, 3d stereo imaging of abnormal waves in a wave basin, Proceedings OMAE (2015) 2015-42318.

[29] T. Waseda, T. Kinoshita, L. Cavaleri, A. Toffoli, Third-order resonant wave interactions under the influence of background current fields, Journal of Fluid Mechanics 784 (2015) 51-73.

[30] C. Kharif, E. Pelinovsky, A. Slunyaev, Rogue waves in the ocean. Advances in Geophysical and Environmental Mechanics and Mathematics, Springer, Berlin, 2009.

[31] K. B. Dysthe, Note on a modification to the nonlinear Schrödinger equation for application to deep water waves, Proceedings of the Royal Society of London. A. Mathematical and Physical Sciences 369 (1736) (1979) 105-114.

[32] K. Trulsen, C. T. Stansberg, Spatial evolution of water surface waves: Numerical simulation and experiment of bichromatic waves, in: The Eleventh International Offshore and Polar Engineering Conference, International Society of Offshore and Polar Engineers, 2001.

[33] E. Kit, L. Shemer, Spatial versions of the Zakharov and Dysthe evolution equations for deep-water gravity waves, Journal of Fluid Mechanics 450 (2002) 201-205.

[34] L. Shemer, L. Alperovich, Peregrine breather revisited, Physics of Fluids (1994-present) 25 (5) (2013) 051701.

[35] A. Chabchoub, B. Kibler, J. M. Dudley, N. Akhmediev, Hydrodynamics of periodic breathers, Phil. Trans. R. Soc. A 372 (2027) (2014) 20140005.

[36] A. Chabchoub, R. H. Grimshaw, The hydrodynamic nonlinear Schrödinger equation: Space and time, Fluids 1 (3) (2016) 23. 
[37] H. Houtani, T. Waseda, K. Tanizawa, Experimental and numerical investigations of temporally and spatially periodic modulated wave trains, Physics of Fluids 30 (3) (2018) 034101.

[38] O. Kimmoun, H. Hsu, H. Branger, M. Li, Y. Chen, C. Kharif, M. Onorato, E. Kelleher, B. Kibler, N. Akhmediev, et al., Modulation instability and phase-shifted fermi-pasta-ulam recurrence, Scientific reports 6 (2016) 28516.

[39] A. Mussot, C. Naveau, M. Conforti, A. Kudlinski, F. Copie, P. Szriftgiser, S. Trillo, Fibre multi-wave mixing combs reveal the broken symmetry of Fermi-Pasta-Ulam recurrence, Nature Photonics 12 (5) (2018) 303.

[40] M. Onorato, D. Proment, G. Clauss, M. Klein, Rogue Waves: From nonlinear Schrödinger breather solutions to sea-keeping test, PLOS ONE 4 (1) (2013) i2bA2.

[41] E. Kartashova, Energy spectra of 2d gravity and capillary waves with narrow frequency band excitation, EPL (Europhysics Letters) 97 (3) (2012) 30004.

[42] E. Kartashova, Energy transport in weakly nonlinear wave systems with narrow frequency band excitation, Physical Review E 86 (4) (2012) 041129.

[43] A. V. Slunyaev, V. I. Shrira, On the highest non-breaking wave in a group: fully nonlinear water wave breathers versus weakly nonlinear theory, Journal of Fluid Mechanics 735 (2013) 203248.

[44] A. Slunyaev, A. Dosaev, On the incomplete recurrence of modulationally unstable deep-water surface gravity waves, Communications in Nonlinear Science and Numerical Simulation 66 (2019) 167-182. 